\title{
Vulvar intraepithelial neoplasia and microinvasive carcinoma of the vulva
}

\author{
CH BUCKLEY, EB BUTLER, H FOX \\ From the Departments of Pathology, St Mary's Hospital, Manchester, and University of Manchester, \\ Manchester M13 9PT
}

SUMMARY The pathological, cytological, and clinical features of vulvar intraepithelial neoplasia (VIN) are described. The rate of progression of VIN III to an invasive carcinoma is very low and spontaneous regression can occur. These features prevent the drawing of a direct analogy between vulvar and cervical intraepithelial neoplasia.

The concept of microinvasive carcinoma of the vulva is discussed, and it is concluded that no satisfactory definition of this entity has been achieved.

\section{Vulvar intraepithelial neoplasia}

There is an increasing tendency to group all cases of intraepithelial squamous cellular atypia in the vulva under the single heading of vulvar intraepithelial neoplasia (VIN) and to discontinue the use of terms such as "mild atypia," "moderate atypia," and "carcinoma in situ." " Both Paget's disease and melanoma in situ are considered by some to fall within the category of VIN, but the importance of these lesions is quite different from that of squamous intraepithelial neoplasia and in our view the term VIN should be restricted to vulvar squamous cell abnormalities.

The adoption of the VIN terminology is partly based on an analogy with cervical intraepithelial neoplasia (CIN), and all the arguments which have been put forward for the adoption of the CIN terminology ${ }^{2}$ apply with equal force to the use of the VIN nomenclature. The single most important factor responsible for the introduction of the VIN terminology, however, has been the finding that in most cases of mild or moderate atypia the abnormal cells are aneuploid ${ }^{3}$; there is no correlation between the degree of abnormality within the vulvar squamous epithelium and ploidy values. Most examples of vulvar carcinoma in situ $(92 \%)$ are also aneuploid ${ }^{4}$ and there is no evidence that aneuploid atypia and

Accepted for publication 25 July 1984

*This review is an expanded version of a paper given by Professor Fox to a course on vulvar disease in Barcelona in 1983; the original paper will be published, in Spanish, in the published proceedings of that course. carcinomas in situ differ biologically from each other in any way apart from the degree of maturation, or differentiation, of the intraepithelial lesion.

VIN may arise in the setting of a hyperplastic or mixed dystrophy, may develop in condylomata, and may occur in otherwise normal vulvar skin. This latter possibility is often discounted, but some cases of VIN are not obviously associated with either a dystrophic or condylomatous lesion. It has been claimed that VIN can develop in lichen sclerosus; this has not been our experience, although the two conditions may coexist.

\section{HISTOLOGICAL FEATURES OF VIN}

Atypia within vulvar squamous epithelium is manifest by disturbances of normal epithelial stratification and abnormalities of cellular maturation. Two basic patterns are seen: one in which atypical cells of basal or parabasal type extend into the upper layers of the epidermis and the other in which premature cellular maturation occurs, often in association with epithelial multinucleation, corps ronds, and koilocytosis. Common to both forms of atypia are the presence of mitotic figures, sometimes of abnormal or bizarre form, above the basal layers of the epithelium, cellular and nuclear pleomorphism, a high nucleocytoplasmic ratio, irregular clumping of nuclear chromatin, and, in many cases, either parakeratosis or hyperkeratosis.

When cellular abnormalities, together with lack of stratification, are limited to the lower third of the vulvar squamous epithelium (Fig. 1) the lesion is 


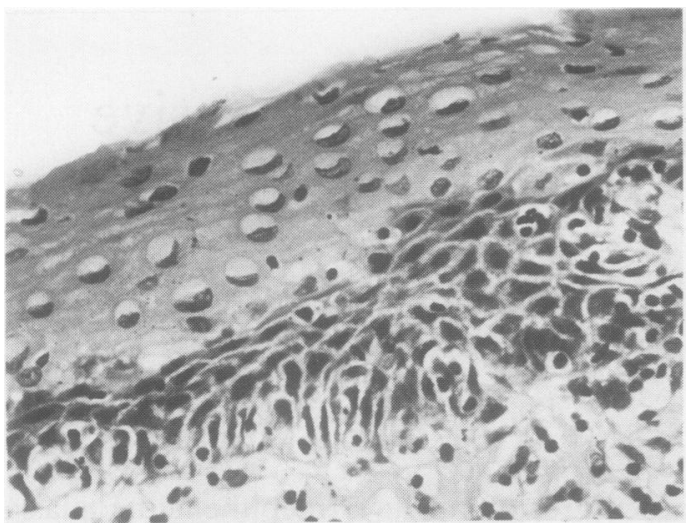

Fig. 1 Vulvar intraepithelial neoplasia (VIN I). Abnormal cells are limited to the lower third of the epithelium. Note the koilocytes in the upper strata. Haematoxylin and eosin. $\times$ 420.

classed as VIN I, which corresponds to mild atypia; extension of abnormal cells into the middle third of the epithelium (Fig. 2) puts the lesion into the category of VIN II, which is equivalent to moderate atypia. Involvement of the upper third of the epithelium leads to a diagnosis of VIN III, a category encompassing both severe atypia and carcinoma in situ. Grading of the form of VIN in which

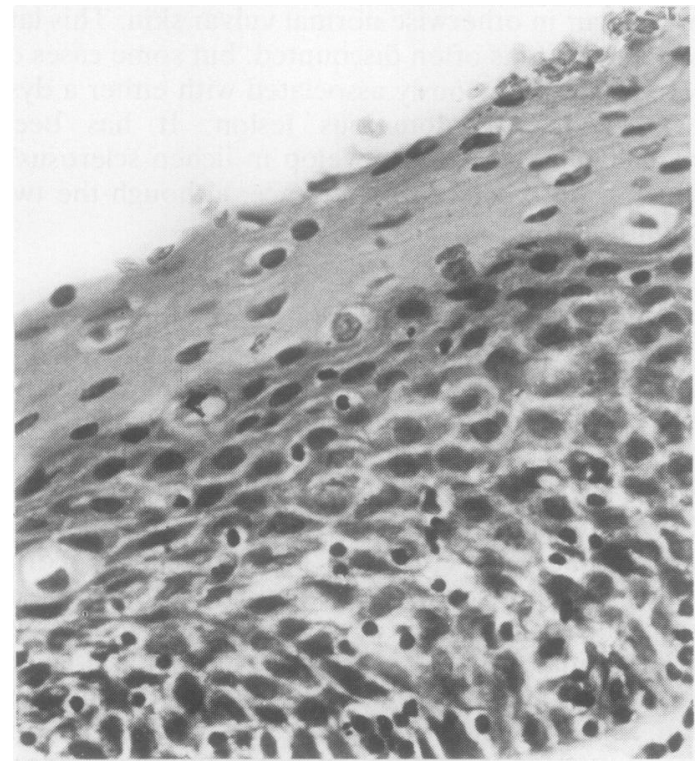

Fig. 2 Vulvar intraepithelial neoplasia (VIN II). Abnormal cells occupy the lower two thirds of the epithelium. This is from the same patient as the tissue shown in Fig. 1 and it emphasises the variation in the grade of VIN seen in a single specimen. Haematoxylin and eosin. $\times 420$.

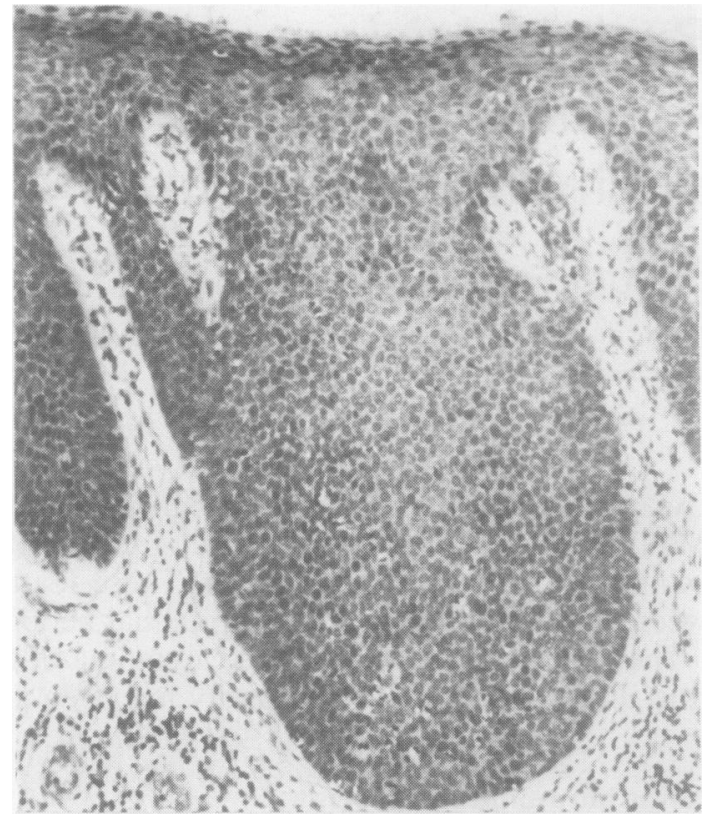

Fig. 3 Vulvar intraepithelial neoplasia (VIN III). The entire epithelium is replaced by cells of basaloid type. Haematoxylin and eosin. $\times 150$.

basal or parabasal type cells extend into the upper epithelium, the basaloid type of VIN (Fig. 3), is relatively easy, but grading of the form of VIN charac-

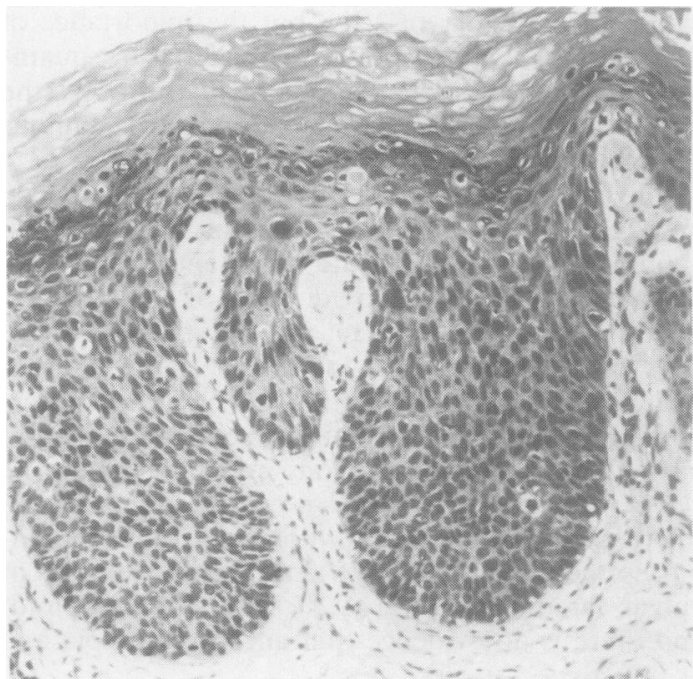

Fig. 4 Vulvar intraepithelial neoplasia. (Bowenoid VIN III). There is some preservation of cellular stratification but cellular pleomorphism and individual cell keratinisation are present. In this area there is hyperkeratosis. Haematoxylin and eosin. $\times 150$. 


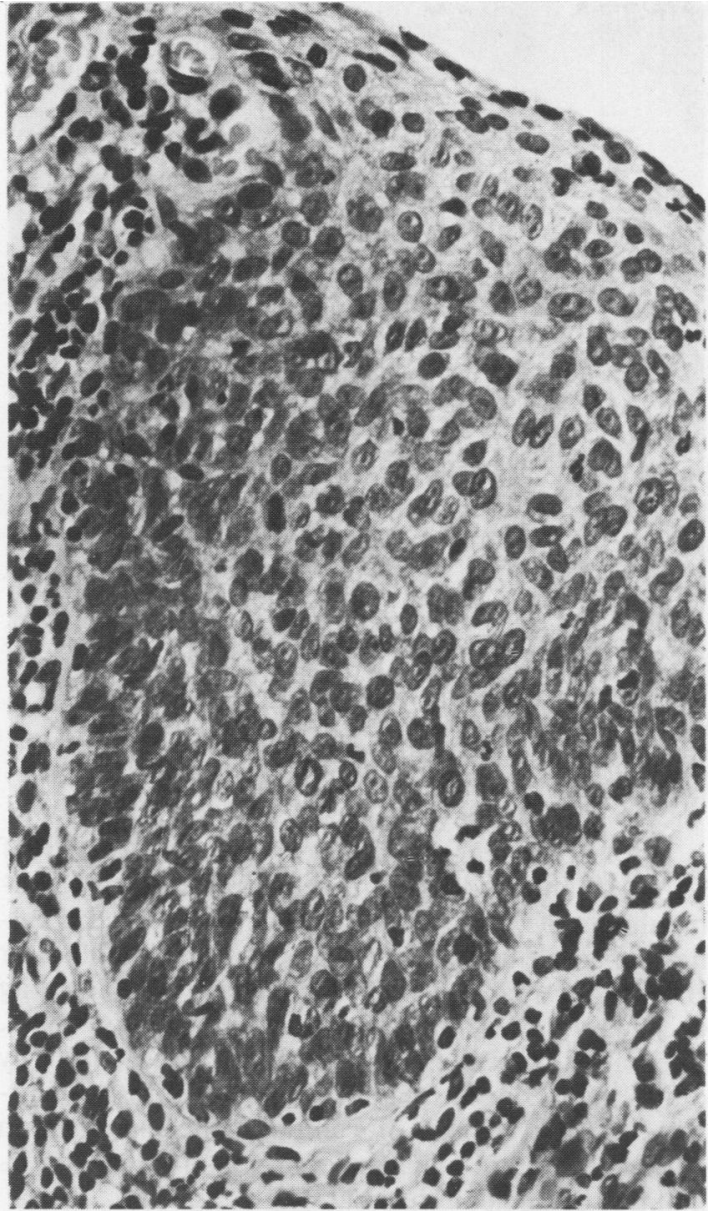

Fig. 5 VIN III. The epithelium is occupied by closely packed cells with high nucleocytoplasmic ratios and there is parakeratosis. Haematoxylin and eosin. $\times 400$.

terised by premature cellular maturation, often with individually keratinised cells lying deep within the epidermis, is more difficult. This form of VIN, often known as the Bowenoid type (Fig. 4), is characterised by a much greater degree of cellular maturation and cellular stratification than is seen in the basaloid form, while cellular and nuclear pleomorphism is often pronounced. It is accepted, however, that grading of VIN is largely arbitrary and subjective, and these three categories of VIN may not directly correspond to increasing risks of invasive carcinoma. Other factors, such as the age of the patient, the area of the lesion, the immune status of the patient, and the type of clinical presentation should also be taken into consideration.

A clear distinction is usually drawn between the basaloid type of VIN III, which was previously

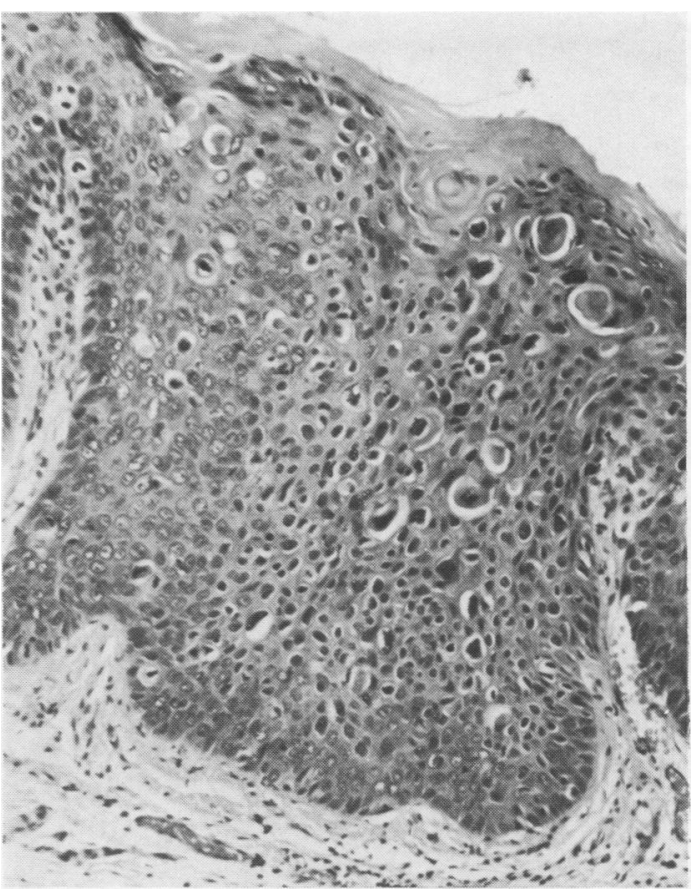

Fig. 6 Bowenoid VIN III. The epithelium is acanthotic and hyperkeratotic. Premature cytoplasmic maturation, pleomorphism, and koilocytes are conspicuous.

Haematoxylin and eosin. $\times 150$.

regarded as the more common type, and Bowenoid VIN III, which, in our experience, has increased in prevalence in latter years and now constitutes the greater proportion of our material.

In the basaloid form of VIN III a parakeratotic layer overlies an epithelium which is otherwise occupied throughout its entire thickness by closely packed, non-stratified cells, which show nuclear crowding and a high nucleocytoplasmic ratio (Fig. 5). In the Bowenoid form, premature maturation, variable retention of stratification, and pleomorphism are key features (Fig. 6). Often, however, in certain areas of such lesions, the specific features are fewer and the epidermis is composed of large pleomorphic cells with relatively scanty cytoplasm in which there are frequent mitoses (Fig. 7). It is not uncommon to find lesions which have a basaloid pattern in some areas and the alternative pattern in others; the two forms are therefore not mutually exclusive. It remains worthwhile to continue to differentiate them, however, largely because Bowenoid VIN III is typically found in patients with current or previous evidence of papilloma virus infection, having either flat or papillary condylomata in the lower genital tract. 


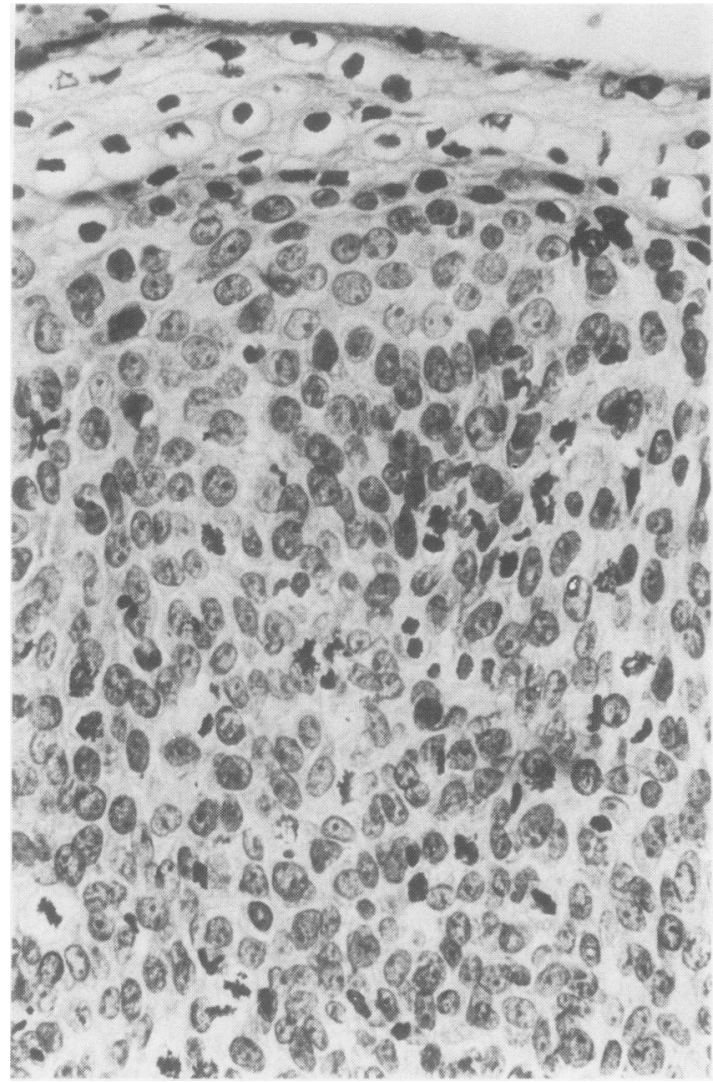

Fig. 7 VIN III. In this area, which is from the same patient as the tissue shown in Fig. 6, the atypical cells are large and there are frequent mitoses. Koilocytes are again present in the superficial layers. Haematoxylin and eosin. $\times 420$.

In many cases of VIN III intraepithelial or intradermal melanin, or both, is present, and this is occasionally a prominent feature (Fig. 8). The pigmented forms of VIN are sometimes regarded as a separate entity-Bowenoid pigmented papulosis-but pigmentation is not limited to this form of intraepithelial neoplasia and the presence of pigment laden dermal macrophages is not specific to VIN, merely indicating the presence of pigmentary incontinence in the vulvar epithelium. There seems little justification, therefore, for regarding the pigmented form of VIN as a separate condition.

A further lesion which is often regarded as a variant of VIN III $^{6}$ is that in which there is little evidence of surface epithelial atypia, the principal abnormality being in the base of the rete ridges where intraepithelial "pearl" formation is seen (Fig. 9). Whether or not this abnormality truly merits inclusion within the VIN category remains a matter for further study.

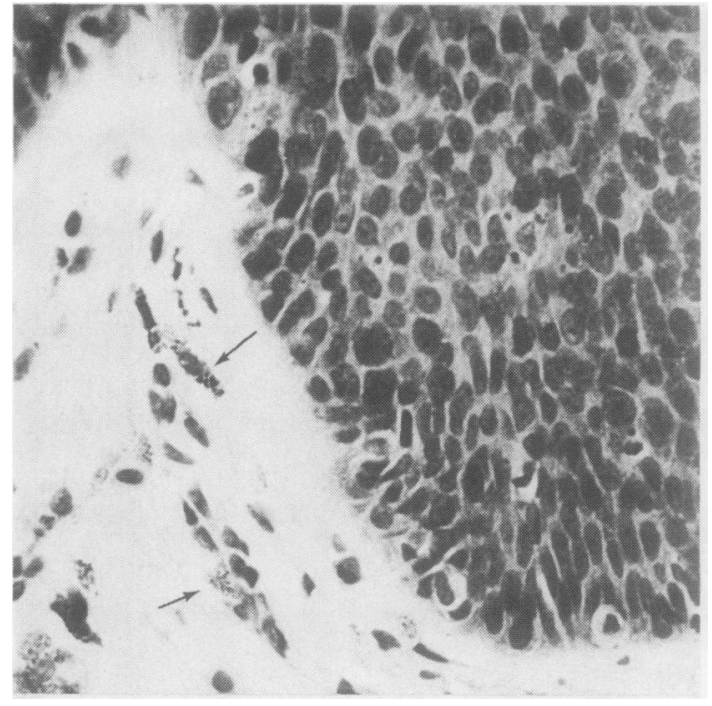

Fig. 8 VIN III. Pigmentary incontinence is indicated (arrow) by the presence of pigment laden dermal macrophages. Haematoxylin and eosin. $\times 375$.

The histological diagnosis of VIN, particularly of VIN III and especially of the basaloid or parabasaloid type, is often straightforward but difficulties may arise in distinguishing examples of VIN I or II from the reactive cytological changes which may occur in a variety of vulvar inflammatory disorders. The latter, however, are usually characterised by only a minor degree of cellular pleomorphism, a variable degree of loss of cellular cohesion, and an absence, despite nuclear enlargement, of abnormalities of nuclear chromatin dispersal.

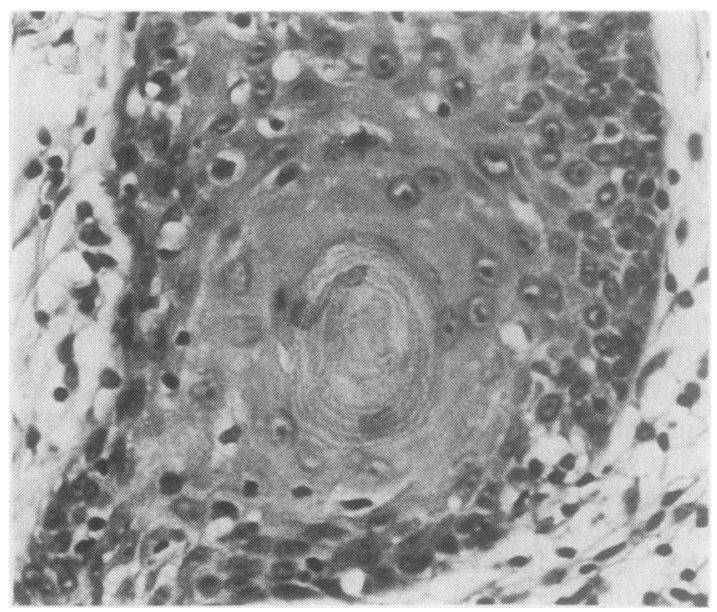

Fig. 9 VIN III. An epithelial pearl in the base of a rete ridge. Haematoxylin and eosin. $\times 420$. 


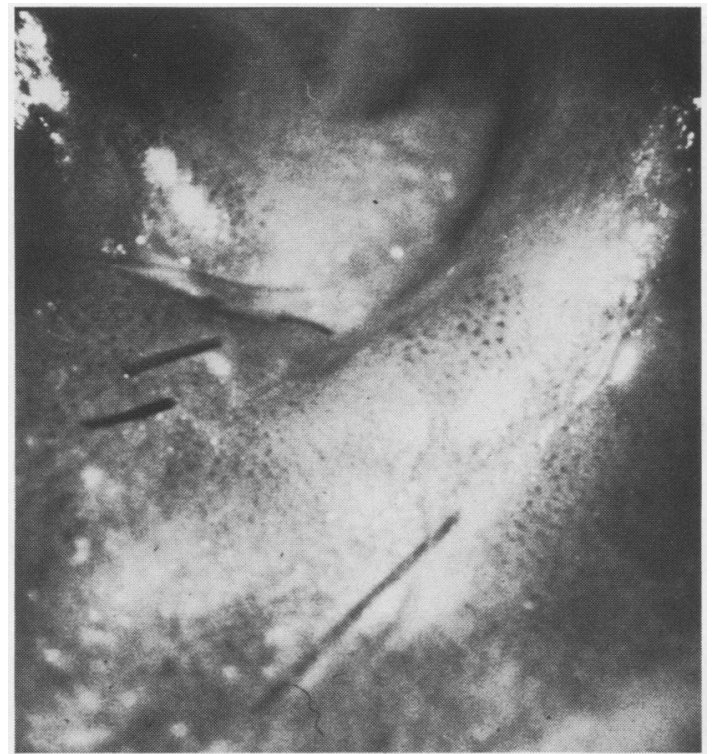

Fig. 10 Colposcopy of vulva. This illustrates a discrete area of VIN III to the right of the introitus. The posterior vaginal wall is seen to bulge in the top of the picture. Note patches of hyperkeratosis with areas showing punctuation between them. Saline. $\times 16$.

\section{COLPOSCOPIC AND CYTOLOGICAL FEATURES}

Cytological evaluation of the degree of abnormality in cases of VIN is less reliable than in the study of cervical epithelial lesions. This is largely because throughout the whole range of vulvar epithelial abnormalities a surface layer of hyperkeratosis often prevents exfoliation of cells. Collection of cytological specimens under colposcopic control gives a better yield of cells, for it then becomes possible to recognise areas without hyperkeratosis and with abnormal vascular patterns.

In some cases, however, colposcopy reveals only a thick white epithelium due to hyperkeratosis or a skin which appears either normal or injected as a result of scratching and infection. Colposcopy and cytology have nothing to offer in such cases and random biopsies are needed to establish a diagnosis.

In other cases of VIN vascular areas showing punctuation, a mosaic pattern, or vascular irregularity may be recognised colposcopically; these patterns reflect changes in abnormal epithelium in the same way as they do in the cervix (Fig. 10). Such areas are usually sharply demarcated from normal skin and a cytological scrape specimen commonly yields abnormal cells. These are also areas which should be biopsied.

The cytological criteria for recognising dyskaryotic cells, shed from areas of VIN, and malignant cells, shed from invasive vulvar neoplasms, have been described previously in relation to cervical epithelial abnormalities. ${ }^{2}$ These are a less reliable guide to the degree of abnormality than is the case with cervical lesions, however, and this may be related to differing rates of exfoliation and to cellular reactions due to trauma or infection. Two main cell patterns emerge. In the first the smear is scanty and contains well differentiated, often keratinised, cells; nuclei may be large and degenerate with loss of recognisable chromatin pattern or hyperchromatic with a coarsely granular chromatin pattern similar to that seen in dyskaryotic cells in a cervical smear. The maturity of the dyskaryotic cells would point to a diagnosis of VIN I or II, but this is often an underestimate as the cells in the smear come from a thick layer of parakeratosis which may overlie a VIN III. Careful search may reveal occasional poorly differentiated dyskaryotic cells when vessels bring deeper epithelium near the surface (Fig. 11a and $b$ ). The second pattern is seen in the absence of pronounced hyperkeratosis and parakeratosis and is characterised by a more profuse exfoliation of cells. This pattern is more common in VIN III of the small cell (basaloid) type (see Fig. 3) and cells are immature with scanty cytoplasm. Chromatin patterns range from that typical of dyskaryotic nuclei to nuclei which show irregular areas of clearing, sharp irregularities of the nuclear membrane, and prominent irregular nucleoli-that is, nuclei of the malignant type which suggest the presence of an invasive lesion (Fig. 12). Cells of this type are indeed seen in cases of early invasion, but more commonly biopsy of vulvar lesions from which these cells have been shed shows only VIN III. It is probable that the confusion results from a pronounced reaction in dyskaryotic cells due to infection and trauma.

\section{CLINICAL ASPECTS}

The clinical features of VIN I and VIN II are poorly documented and virtually all the comments made here refer only to cases of VIN III. The incidence of VIN III is low, but there seems little doubt that this lesion is being encountered much more often than previously, particularly in relatively young women..$^{6-9}$ Thus, for example, only 14 cases of VIN III were diagnosed at the Johns Hopkins Hospital in Baltimore in 1958 while 122 cases were seen during $1978 .^{\circ}$ Similarly, in Buenos Aires the incidence of VIN III has risen from one case every nine years between 1928 and 1967 to one case a year during the period 1968-76 and to four cases a year during the period $1978-81 .^{10}$ To some extent this increased incidence may reflect heightened awareness of the condition and a greater readiness to diagnose the lesion, but nevertheless it appears that most of the 


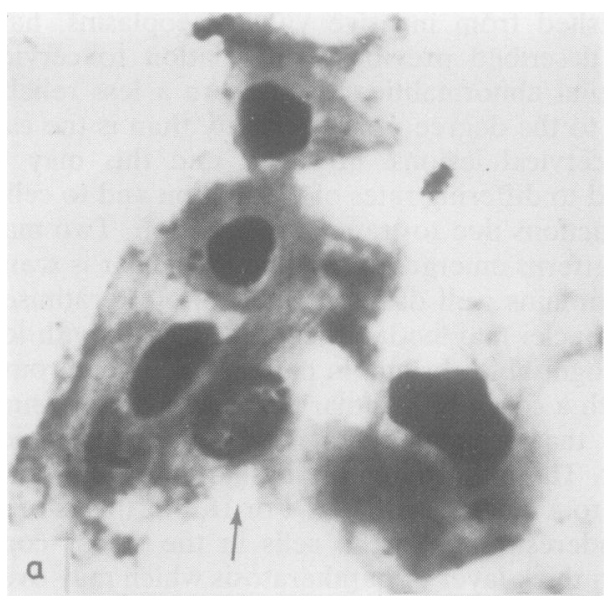

Fig. 11(a) Vulvar smear. Cells are seen with large pyknotic nuclei and dense cytoplasm. This is a common finding when parakeratosis is present which prevents exfoliation of poorly differentiated dyskaryotic cells which would more truly reflect the degree of abnormality. In this field one immature dyskaryotic cell is seen (arrow). Papanicolaou's stain. Original magnification $\times 280$. (b) Tissue. This field shows the surface layers of a section diagnosed as VIN III. The cells are similar to those in (a). Failure of differentiation is seen in the lower part of the field. Haematoxylin and eosin. Original magnification $\times 280$.

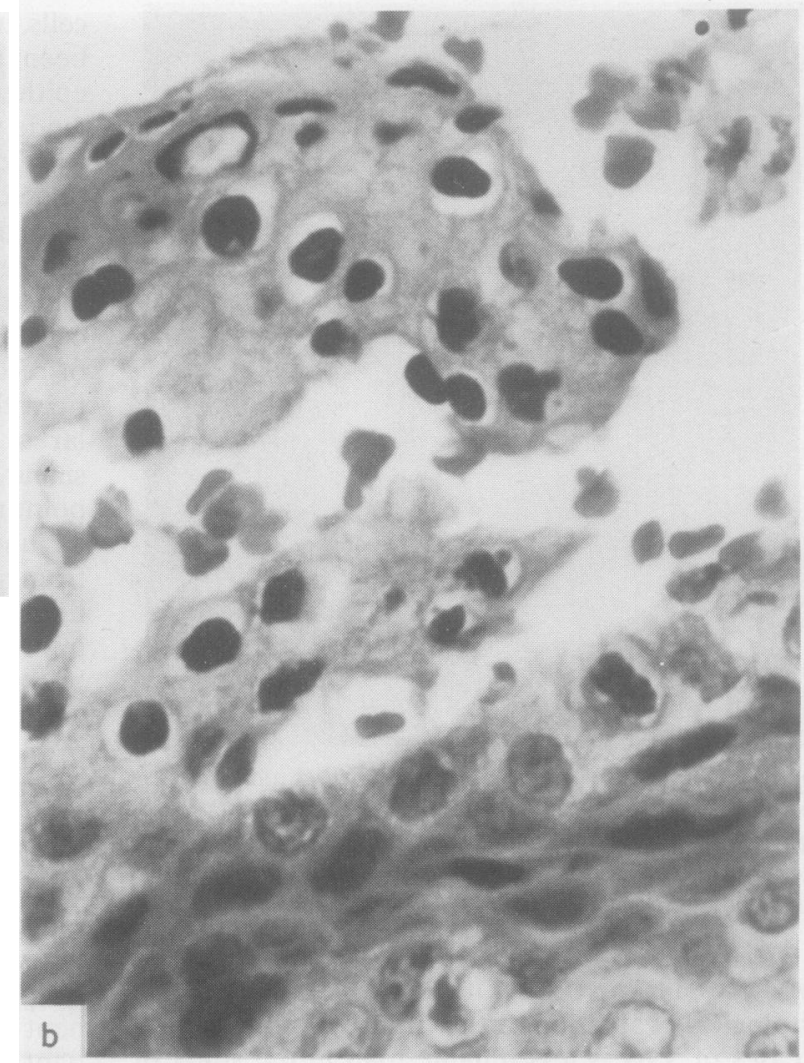

under 40 years has been in the region of $40 \% .4691112$

There is a clear association between VIN III and both sexually transmitted diseases and neoplastic lesions elsewhere. Thus about $20-25 \%$ of patients with VIN III have concurrent, past, or subsequent CIN, 68-1012 13 while a substantial proportion, between 8 and $40 \%$, have concurrent or subsequent invasive neoplasms either in the genital tract or elsewhere in the body. ${ }^{412-14}$ Friedrich $e t$ al ${ }^{4}$ noted that the overall incidence of sexually transmitted diseases in their series of 50 women with VIN III was $60 \%$, which is a notably higher incidence than previously cited ones of $22 \%{ }^{14}$ and $38 \% .^{11}$ Whether this increasing association between VIN III and sexually transmitted diseases is a true one or simply represents population bias remains to be resolved. A history of herpes vulvitis is obtained from about $10-12 \%$ of women with VIN III, while condylomata acuminata are present in between 15 and $30 \% .4691112$ Bernstein et al ${ }^{12}$ noted that condylomata are found almost entirely in patients under 40 years of age and usually occur in association with the multifocal form of VIN III.

The most common presenting complaint of
Fig. 12 Vulvar smear. Undifferentiated dyskaryotic cells shed from VIN III of the small cell (basaloid) type. Note the uniformity of cell size with some nuclei showing irregularities of outline, clear areas in the nuclear chromatin pattern, and prominent nucleoli. cf. Fig. 3. Papanicolaou's stain. Original magnification $\times 280$. 
women with VIN III is pruritus, but about a third have noted some atypicality of the vulvar skin while a substantial proportion, ranging in different series from 18 to $46 \% \%^{4691012}$ are asymptomatic; in these latter patients the vulvar lesion is usually detected incidentally during treatment for sexually transmitted disease or CIN. Macroscopically, the lesions of VIN III may be discrete and sharply localised or may affect the entire vulva; about $70-75 \%$ of cases are multifocal, while spread to involve the perianal skin occurs in a quarter of patients. The lesions are often raised above the level of the surrounding skin and may have a somewhat roughened appearance or be smooth; they can be white, grey, pink, dull red, or brown and some are darkly pigmented. The diagnosis is established only by biopsy and the tendency towards a multicentric growth pattern makes multiple sampling of the vulvar skin mandatory.

\section{NATURAL HISTORY AND TREATMENT}

Very little is known about the natural history of VIN I and II and the risk of such lesions eventually evolving into an invasive carcinoma has not been accurately determined; such a risk is, however, probably low. ${ }^{7}$ The natural history of VIN III is only just beginning to emerge, but it is already clear that the biological behaviour of VIN III is not directly comparable with that of CIN III. Thus the incidence of progression to a frankly invasive neoplasm is low. In one series of 106 women with VIN III, only four progressed to an invasive lesion ${ }^{6}$ while in another of 37 patients only one developed an invasive carcinoma. ${ }^{4}$ There is certainly no doubt that VIN III can advance to an invasive squamous cell carcinoma, ${ }^{14-16}$ but it is equally clear that progression to this stage is the exception rather than the rule. In most reported cases in which VIN III has progressed to an invasive carcinoma the patient has either been elderly-that is over 75 years-or has been immunosuppressed, and examples of progression in young non-immunosuppressed patients are, though recorded, ${ }^{17}$ extremely unusual. By contrast, cases of untreated VIN III may undergo spontaneous regression. Friedrich et $a^{4}$ followed nine patients who were, for various reasons, not offered any treatment for their VIN III and noted that in five of these cases the lesion underwent spontaneous regression. Bernstein et al $^{12}$ followed up 13 untreated women with VIN III and recorded spontaneous regression of the lesion in five of these cases within six months. In the series of Friedrich et $\mathrm{ll}^{4}$ patients whose lesions underwent spontaneous regression were young, had multicentric disease, and were, in four of the five cases, pregnant at the time of diagnosis. Women undergoing spontaneous regression of VIN III in the series of Bernstein et al ${ }^{12}$ were also young (aged under 35 years) but only three of the five patients had multicentric lesions and none was pregnant at the time of diagnosis. The fact that some cases of VIN III undergo spontaneous regression may suggest that these are not true examples of intraepithelial neoplasia, but all the lesions showing this change in the series of Friedrich et al $^{4}$ were aneuploid at the time of diagnosis and all eventually reverted to a normal ploidy.

In view of the low rate of advance of VIN III to invasive carcinoma there has been an increasing tendency towards more conservative treatment and radical vulvectomy is not indicated for these patients. ${ }^{18}$ Current treatment tends towards either local excision or laser therapy for localised lesions and skinning vulvectomy for young patients with extensive multicentric disease. A case may be made for partial or total vulvectomy in elderly women with extensive multifocal disease. The use of topical 5-fluorouracil has proved disappointing but cryosurgery has met with some success when used on small unifocal lesions. The one great advantage of surgical treatment of VIN III is that it allows a complete histological survey to be carried out and foci of early invasion to be recognised.

Irrespective of the type of treatment employed there is a recurrence rate of about $10 \%$ : recurrent lesions usually respond well to repeat therapy and rarely progress to an invasive neoplasm.

\section{Microinvasive carcinoma of the vulva}

Microinvasive carcinoma is an entity which is fully accepted, though still variably defined, by students of cervical pathology and it has appeared logical to try to extrapolate this concept of an early form of invasive carcinoma to the vulva. The difficulties which have been encountered in attempting to define a microinvasive carcinoma of the cervix pale almost into insignificance when compared with those met with in trying to achieve an acceptable definition of a microinvasive carcinoma of the vulva. Currently, the situation is confused, with some workers believing that a definite microinvasive stage of vulvar carcinoma can be recognised and others maintaining that any attempt to draw an analogy between cervical and vulvar lesions is misguided and that a vulvar carcinoma is either invasive or not. ${ }^{19}$ The inherent complexity of the problem has been confounded by a number of factors, which include differing concepts of a microinvasive carcinoma, the lack of an agreed definition, the use of imprecise pathological data, and the accumulation of conflicting findings. It should also be mentioned that the picture has not been made any clearer by the use of 
a variety of terms; thus, "superficial infiltrating carcinoma," "superficially invasive carcinoma," "occult carcinoma," "early vulvar cancer," "early invasive carcinoma," "stage 1a carcinoma," and "microinvasive carcinoma" have often been used synonymously and interchangeably, and it is sometimes far from certain whether those using these terms are all referring to the same entity or not.

The most widely accepted definition of a microinvasive carcinoma of the vulva, and one which is employed in the current standard text on gynaecological oncology, ${ }^{20}$ is: "A squamous cell carcinoma $2 \mathrm{~cm}$ or less in diameter with no more than $5 \mathrm{~mm}$ stromal invasion where the depth of invasion is the maximum measured in any one high power field. The presence of confluence, vascular channel permeation or cellular anaplasia does not exclude the case from this category."

It will be noted that central to this definition is the considerable emphasis placed on a precisely measured depth of stromal invasion by the tumour. The maximum depth of $5 \mathrm{~mm}$ has been based on the report of Wharton et al ${ }^{21}$ that 25 patients with vulvar carcinomas invading to a depth of $5 \mathrm{~mm}$ or less had no nodal metastases or recurrence of tumour and all survived, while of 20 women whose neoplasms invaded beyond this level five had lymph node spread and three died of vulvar cancer. Wharton et al were, however, at pains to emphasise that the limit of $5 \mathrm{~mm}$ had been arbitrarily chosen and pointed out that further experience was required to determine whether or not this figure adequately characterised a microinvasive stage. Their doubts on this point were fully justified for it has since become clear that about $12 \%$ of patients with tumour invasion to a depth of $5 \mathrm{~mm}$ or less have inguinal node metastases at the time of vulvectomy. ${ }^{22}$ The only truly valid concept of a microinvasive carcinoma is of one which, though seen histologically to have broken out from the confines of the epithelium, is only invading the stroma to an extent as to carry no risk of lymph node metastasis. Therefore a definition employing $5 \mathrm{~mm}$ as a maximum depth of invasion is insufficiently stringent and is associated with an unacceptably high incidence of nodal involvement. There has therefore been an increasing tendency for a cut off point of $3 \mathrm{~mm}$ to be used for defining a microinvasive lesion. ${ }^{23-26}$ Even this may be too lenient a figure, however, for there have been patients with tumours which though only invading to a depth of between 1 and $2 \mathrm{~mm}$ were associated with lymph node metastases either at the time of, or subsequent to, initial diagnosis, ${ }^{27-31}$ and some of these women eventually died of widespread disease. It has become apparent that it is only those tumours which invade to a depth of $1 \mathrm{~mm}$ or less which are not associated with any risk of lymph node involvement, ${ }^{24} 3132$ though the risk of such spread is certainly low in carcinomas invading up to $1.5 \mathrm{~mm} .{ }^{22}$ Some go further and suggest that any tumour invading to a depth greater than $0.8 \mathrm{~mm}$ carries a risk of metastasis or recurrence..$^{33} 34$

When considering any statements about the depth of invasion of a vulvar carcinoma we should take into account the fact that there has been no agreement as to the point from which the depth of invasion should be measured. The surface of the tumour, the surface of adjacent intact epithelium, the lower margin of adjacent surface epithelium, the base of the adjacent most superficial dermal papilla, the tip of the deepest adjacent rete ridge, and the superficial granular layer of the overlying epithelium have all been used as starting points for measuring tumour depth, and this makes comparison between different studies invidious. Thus, for example, Wilkinson $e a^{22}$ used the base of the adjacent most superficial dermal papilla as their point from which tumour depth was measured and concluded that nodal metastasis was extremely unlikely if the depth of invasion was less than $1.5 \mathrm{~mm}$. By contrast, Woodruf ${ }^{34}$ considered that depth of tumour invasion of more than $0.8 \mathrm{~mm}$ was potentially dangerous but he was measuring this distance from the tip of the deepest rete ridge. It is obviously difficult to compare these two studies as they are based on differing criteria, and it is even more difficult to interpret those far from uncommon studies in which no details are given about the point from which depth of invasion was measured. There is probably no single site from which tumour invasion should be measured which has incontrovertible advantages over all other starting points, though the surface of the adjacent epithelium is least satisfactory because of variations in the keratin layer while the superficial granular layer appears an unsuitable starting point if only because it is sometimes absent from vulvar skin. Until there is a measure of agreement as to the point from which depth of invasion is measured all comments about the importance of particular levels of invasion become almost meaningless.

Should emphasis be placed on depth of invasion as a paramount defining feature of a microinvasive carcinoma? Burghardt ${ }^{35}$ has argued that it is tumour volume rather than depth of tumour invasion which is most clearly and consistently related to the risk of nodal metastases and has pointed out that depth of invasion of a tumour is only of real importance when, in sites such as the gastrointestinal tract, tissue boundaries are crossed and there is invasion from a lymphatic free zone to an area which contains lymphatics. In the vulva increasing depth of invasion does not involve the breaching of any anatomical 
barriers, and the available evidence suggests that lymphatic vessels must lie within 1-2 $\mathrm{mm}$ of the basement membrane of the surface epithelium. Very little is known about the volume of microinvasive tumours of the vulva, though there is no clear correlation between depth of invasion and tumour mass. It is true that tumour volume remains below 1000 $\mathrm{mm}^{3}$ when the depth of invasion is less than $1.5 \mathrm{~mm}$, but extension beyond that point is associated with a wide range of tumour volumes that do not relate in a linear manner to the depth of invasion. ${ }^{22}$

Even a tumour volume of $1000 \mathrm{~mm}^{3}$ appears to be too large to fall within the category of a microinvasive lesion, and this large mass reflects, to a certain extent, the defining feature of a maximum diameter of $2 \mathrm{~cm}$. It has been suggested that the maximum diameter of a microinvasive carcinoma should be only $1 \mathrm{~cm},{ }^{36}$ but Magrina et al $^{24}$ noted that $10 \%$ of patients with tumours measuring $1 \mathrm{~cm}$ or less in diameter developed nodal metastases while Kneale et $a^{20}$ found a $4 \%$ incidence of lymph node involvement and a $16 \%$ incidence of local recurrence in association with neoplasms of $1 \mathrm{~cm}$ or less in diameter. Hoffman $e a^{26}$ have argued that tumour diameter correlates with the risk of nodal spread but only because the diameter of a neoplasm is closely related to its depth of invasion. They considered that variable depth was a better predictor of lymph node involvement than diameter and that a knowledge of tumour diameter did not add any further precision to the accuracy of prediction once tumour depth was known.

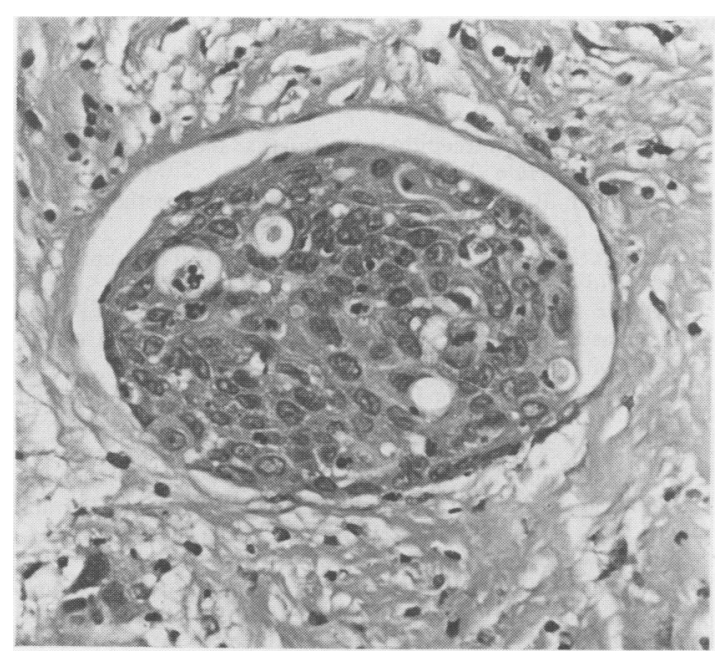

Fig. $13 A$ vascular like space in the dermis distended by the presence of rather poorly differentiated squamous carcinoma. Haematoxylin and eosin. $\times 375$.
Other controversial aspects of the definition of a microinvasive carcinoma of the vulva include the importance or otherwise of features such as vascular space involvement, confluence, and degree of tumour differentiation and the significance of associated VIN.

In some studies invasion of vascular like spaces (Fig. 13) has been significantly associated with an increased risk of nodal metastases: ${ }^{26283738}$ the most striking association was noted in the series of Kolstad et al, ${ }^{31}$ in which $40 \%$ of patients in whom vascular like space invasion was found had nodal metastases while only $3 \%$ of those without tumour involvement of these spaces showed evidence of lymph node spread. By contrast, however, others have been unable to show any clear cut relation between the findings of vascular like space permeation and an increased risk of nodal metastases. ${ }^{22} 33$ Two points should be borne in mind when considering these conflicting reports: firstly, it can be a difficult, subjective and arbitrary decision as to whether tumour cells are within a true vascular like space or within a tissue cleft, and, secondly, the true incidence of vascular like space involvement in vulvar lesions of this type has not been assessed by systematic serial sectioning, a technique which, if findings in the cervix are relevant, could be expected to yield a much higher incidence of vascular like space permeation than that which is currently being reported.

Microinvasive carcinomas of the vulva have been divided into large cell keratinising, large cell nonkeratinising, and small cell non-keratinising types, but it has not been shown that this subdivision is of any prognostic value. ${ }^{24} 263134$ The prognostic importance of tumour differentiation is, however, more contentious: in a number of studies the degree of tumour differentiation has shown no association with the risk of nodal metastases, ${ }^{24-26313437}$ but others have considered poor tumour differentiation as an extremely important predictor of a high risk of lymph node metastases. ${ }^{38} 38$ To some extent this difference of opinion may be simply a reflection of the small number of poorly differentiated neoplasms in any single series of microinvasive vulvar carcinomas.

The importance of confluence of the invading tumour cells has also been a subject for debate. To some extent this is because an opinion as to whether a tumour is showing a confluent pattern or not is often highly subjective. Attempts have been made to define confluence either as "a group of tumour cells filling an area of $2 \mathbf{~ m m}$ or more" 24 or as a "mass of carcinoma filling a $1 \mathrm{~mm}$ or greater field". ${ }^{26}$ Clearly, these differing definitions do little to solve the problem of how to assess confluence, but within 


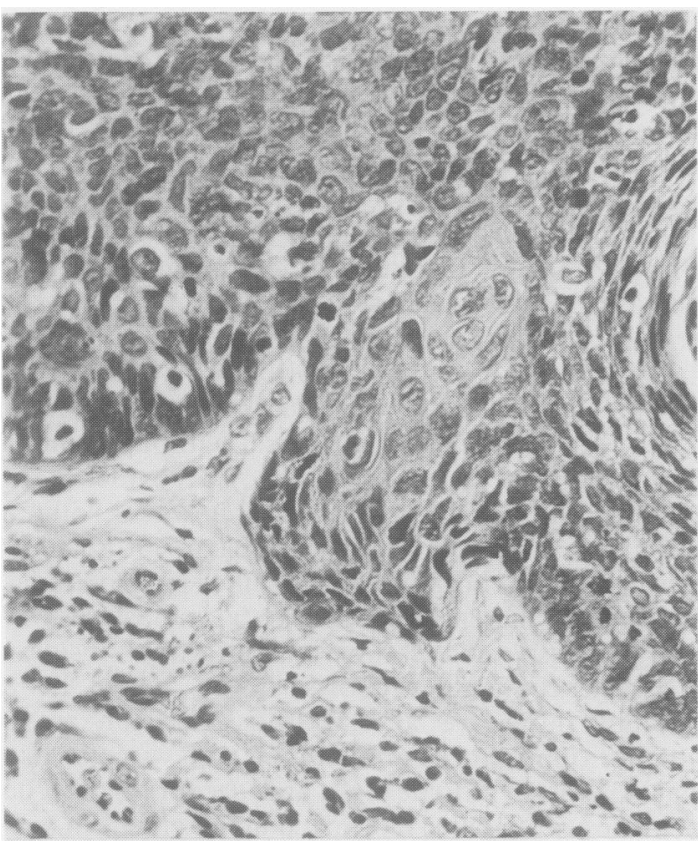

Fig. 14 VIN III with minimally invasive squamous carcinoma. A tongue of cells in which there is a greater degree of cytoplasmic maturation than in the adjacent intraepithelial neoplasm is infiltrating stroma. Haematoxylin and eosin. $\times 375$.

the limits imposed by this difficulty Barnes $e t a^{30}$ and Hoffman $e t a^{26}$ considered that this pattern of growth (a "spray like" pattern) was clearly associated with a high risk of nodal metastases. Magrina et $a l^{24}$ showed that confluence was associated with a worse prognosis but not specifically with an increased risk of nodal spread, while Wilkinson $e t$ $a l^{22}$ found no correlation between a confluent growth pattern and lymph node involvement.

Some workers have found that a microinvasive carcinoma which is arising from an epithelium showing the changes of VIN has an extremely good prognosis with little risk of nodal metastases, ${ }^{25}{ }^{30}$ but Hacker $e a l^{32}$ noted that five of their seven microinvasive carcinomas associated with nodal metastases arose from an epithelium showing VIN III and considered that the presence or otherwise of VIN was of no prognostic importance.

Thus attempts to define a microinvasive carcinoma of the vulva have not been successful. There has been no agreement as to the maximum depth of invasion, the point from which depth of invasion should be measured, the maximum diameter of the tumour, the importance of vascular like space involvement, the significance of the degree of tumour differentiation, the importance of a confluent growth pattern or the relevance of an associated VIN. Faced with this disarray, and with the high incidence of nodal metastases in patients meeting the most widely accepted current definition of microinvasive carcinoma, the International Society for the Study of Vulvar Disease recently agreed that the term "microinvasive carcinoma of the vulva" is misleading and dangerous when taken with its current definition and recommended that its use be discontinued. The Society also recommended that the designation stage Ia of the vulva be used to describe solitary lesions confined to a maximum of 2 $\mathrm{cm}$ diameter and $1 \mathrm{~mm}$ depth of stromal invasion.

The recommendation that use of the term "microinvasive carcinoma of the vulva" should be discontinued is clearly justified in our present state of knowledge, but this does not mean that all attempts to delineate a minimally invasive lesion which can be treated conservatively should be abandoned. Barnes et $a^{l^{30}}$ characterised a form of superficially invasive vulvar neoplasm which did not appear to be associated with any risk of lymph node metastases: this arose from an epithelium showing the changes of VIN as invading foci, single and multiple, formed of single cords or tongues of cells infiltrating the adjacent stroma to a distance of less than $2 \mathrm{~mm}$ from the limiting basement membrane of the epithelial site of origin (Fig. 14). If the foci were multiple, confluence or joining of these separate tongues of invading cells was not seen and vascular like space permeation was not present. Clearly, this pattern corresponds to that described in the cervix as "early stromal invasion" 40 and it would seem reasonable to suggest that this type of invasive lesion could be treated conservatively.

\section{References}

' Crum CP. Vulvar intraepithelial neoplasia: the concept and its application. Hum Pathol 1982;13:187-9.

${ }^{2}$ Buckley $\mathrm{CH}$, Butler EB, Fox $\mathrm{H}$. Cervical intraepithelial neoplasia. J Clin Pathol 1982;35:1-13.

${ }^{3}$ Crum CP, Fu YS, Levine RU, Richart RM, Townsend DE, Fenoglio CM. Intraepithelial squamous lesions of the vulva: biologic and histologic criteria for the distinction of condylomas from vulvar intraepithelial neoplasia. Am J Obstet Gynecol 1982;144:77-83.

${ }^{4}$ Friedrich EG, Wilkinson EJ, Fu YS. Carcinoma in situ of the vulva: a continuing challenge. Am J Obstet Gynecol $1980 ; 136: 830-8$.

${ }^{5}$ Lavery HA, Pinkerton JHM. Vulval dystrophy: its aetiology and classification. In: Morrow CP, Bonnar J, O'Brien TJ, Gibbons WE, eds. Recent clinical developments in gynecologic oncology. New York: Raven Press, 1983:1-14.

- Buscema J, Woodruff JD, Parmley TH, Genadry R. Carcinoma in situ of the vulva. Obstet Gynecol 1980;55:225-30.

Friedrich EG. Intraepithelial neoplasia of vulva. In: Coppleson M, ed. Gynecologic oncology. Edinburgh: Churchill Livingstone, 1981:303-19.

${ }^{8}$ Benedet JL, Murphy EJ. Squamous carcinoma in situ of the vulva. Gynecol Oncol 1982;14:213-9. 
- Caglar H, Tamer S, Hreshchysmn MM. Vulvar intraepithelial neoplasia. Obstet Gynecol 1982;60:346-9.

10 DiPaola GR, Rueda-Leverone NG, Belardi MG, Vighi S. Vulvar carcinoma in situ: a report of 28 cases. Gynecol Oncol $1982 ; 14: 236-42$.

" Forney JP, Morrow CP, Townsend DE, DiSaia PJ. Management of carcinoma in situ of the vulva. Am J Obstet Gynecol $1977 ; 127: 801-6$.

${ }^{12}$ Bernstein SG, Kovacs BR, Townsend DE, Morrow CP. Vulvar carcinoma in situ. Obstet Gynecol 1983;61:304-7.

${ }^{13}$ Boutselis JG. Intraepithelial carcinoma of the vulva. Am J Obstet - Gynecol 1972;113:733-8.

${ }^{14}$ Collins CG, Roman-Lopez JJ, Lee FYL. Intraepithelial carcinoma of the vulva. Am J Obstet Gynecol 1970;108:1187-91.

is Gardiner SH, Stout FE, Arbogast JL, Huber CP. Intraepithelial carcinoma of the vulva. Am J Obstet Gynecol 1953;65:53949.

16 Jones I, Buntine D. Progression of vulval carcinoma in situ. Aust NZ J Obstet Gynecol 1978;18:274-6.

${ }^{17}$ Buscema J, Woodruff JD. Progressive histobiologic alterations in the development of vulvar cancer: report of five cases. Am J Obstet Gynecol 1980;138:146-50.

18 Jordan JA. Conservative management of vaginal and vulval intraepithelial neoplasia. In: Heintz APM, Griffiths CT, Trimbos JB, eds. Surgery in gynecological oncology. Martinus Nijhoff: The Hague, 1984:1-11.

${ }^{19}$ Douglas CP. Vulvar dystrophies. In: Studd J, ed. Progress in obstetrics and gynaecology, vol 3. Edinburgh: Churchill Livingstone, 1983:199-212.

${ }^{20}$ Kneale BLG, Elliott PM, McDonald IA. Microinvasive carcinoma of vulva: clinical features and management. In: Coppleson M, ed. Gynecologic oncology. Edinburgh: Churchill Livingstone, 1981:320-8.

${ }^{21}$ Wharton JT, Gallager S, Rutledge FN. Microinvasive carcinoma of the vulva. Am J Obstet Gynecol 1974;118:159-62.

${ }^{22}$ Wilkinson EJ, Rico MJ, Pierson EK. Microinvasive carcinoma of the vulva. Int J Gynecol Pathol 1982;1:29-39.

${ }^{23}$ Jafari K, Cartnick FN. Microinvasive squamous cell carcinoma of the vulva. Gynecol Oncol 1976; 4:158-66.

${ }^{24}$ Magrina JF, Webb MJ, Gaffey TA, Symmonds RE. Stage I squamous cell cancer of the vulva. Am J Obstet Gynecol 1979; 134:453-7.

${ }^{25}$ Chu J, Tamimi HK, Ek M, Figgs DC. Stage I vulvar cancer: criteria for microinvasion. Obstet Gynecol 1982;59:716-9.

${ }^{26}$ Hoffman JS. Kumar NB, Morley GW. Microinvasive squamous carcinoma of the vulva: search for a definition. Obstet Gynecol 1983;61:615-8.

${ }^{27}$ DiPaola GR, Gomez-Rueda N, Arrhigi L. Relevance of microinvasion in carcinoma of the vulva. Obstet Gynecol 1975;45:647-9.

${ }^{28}$ Parker RT, Duncan I, Rampone J, Creasman W. Operating management of early invasive epidermoid carcinoma of the vulva. Am J Obstet Gynecol 1975;123:349-54.

29 Yazigi R, Piver MS, Tsukada Y. Microinvasive carcinoma of the vulva. Obstet Gynecol 1978;51:368-70.

${ }^{30}$ Barnes AE, Crissman JD, Schellhas HF, Azoury RS. Microinvasive carcinoma of the vulva: a clinicopathologic evaluation. Obstet Gynecol 1980;56:234-8.

${ }^{31}$ Kolstad P, Iversen T, Abeler V, Aalders J. Microinvasive carcinoma of the vulva - definition and treatment problems. Clinics in Oncology 1982;1;355-62.

${ }^{32}$ Hacker NF, Nieberg RK, Berek JS, et al. Superficially invasive vulvar cancer with nodal metastases. Gynecol Oncol 1983;15:66-77.

${ }^{33}$ Buscema J, Stern JL, Woodruff JD. Early invasive carcinoma of the vulva. Am J Obstet Gynecol 1981;140:563-9.

34 Woodruff JD. Early invasive carcinoma of the vulva. Clinics in Oncology 1982;1:349-54.

${ }^{35}$ Burghardt E. Microinvasive tumours of the female genital tract. Clinics Obstet Gynaecol 1984;11:239-57.

${ }^{36}$ DiSaia PJ, Creasman WT, Rich WM. An alternative approach to early cancer of the vulva. Am J Obstet Gynecol 1979; 133:825-30.

${ }^{37}$ Iversen T, Abeler V, Aalders J. Individualised treatment of Stage I carcinoma of the vulva. Obstet Gynecol 1981;57:85-9.

${ }^{38}$ Husseinzadeh $\mathbf{N}$, Zaino $R$, Nahhas WA, Mortel $R$. The significance of histologic findings in predicting nodal metastases in invasive squamous cell carcinoma. Gynecol Oncol 1983; 16:105-11.

${ }^{39}$ Crissman JD, Azoury RS. Microinvasive carcinoma of the vulva: a report of two cases with regional lymph node metastasis. Diag Gynecol Obstet 1981;3:75-80.

${ }^{40}$ Holzer E. Microinvasive carcinoma of the cervix-clinical aspects, treatment and follow-up. Clinics in Oncology $1982 ; 1: 315-22$.

Requests for reprints to: Professor H Fox, Department of Pathology, Stopford Building, University of Manchester, Manchester M13 9PT, England. 